Published in final edited form as:

Prostate Cancer Prostatic Dis. 2017 March ; 20(1): 1-6. doi:10.1038/pcan.2016.51.

\title{
Active Surveillance for Intermediate Risk Prostate Cancer
}

\author{
Marc A. Dall'Era, MD ${ }^{1}$ and Laurence Klotz, MD2 \\ ${ }^{1}$ Department of Urology, University of California, Davis, Sacramento, CA \\ 2Division of Urology, Sunnybrook Health Sciences Centre, University of Toronto, Ontario, Canada
}

\begin{abstract}
Background-Utilization of active surveillance (AS) for prostate cancer is increasing. Optimal selection criteria for this approach are undefined and questions remain on how best to expand inclusion beyond typical men with very low or low risk disease. We sought to review the current experience with AS for men with intermediate risk features.
\end{abstract}

Methods-Pubmed was queried for all relevant original publications describing outcomes for men with prostate cancer managed with AS. Outcomes for patients with intermediate risk features as defined by the primary investigators were studied when available and compared with similar risk men undergoing immediate treatment.

Results-Cancer specific survival for men managed initially with AS is similar to results published with immediate radical intervention. A total of 5 published AS series describe some outcomes for men with intermediate risk features. Definitions of intermediate risk vary between studies. Men with Gleason 7 disease experience higher rates of clinical progression and are more likely to undergo treatment over time. Intermediate risk men with Gleason 6 disease have similar outcomes to low risk men. Men with Gleason 7 disease appear at higher risk for metastatic disease. Novel technologies including imaging and biomarkers may assist with patient selection and disease surveillance.

Conclusions-The contemporary experiences of AS for men with intermediate risk features suggest that although these men are at higher risk for eventual prostate directed treatment, some are not significantly compromising chances for longer-term cure. Men with more than minimal Gleason pattern 4, however, must be carefully selected and surveyed for early signs of progression and may be at increased risk of metastases. Incorporating information from advanced imaging and biomarker technology will likely individualize future treatment decisions while improving overall surveillance strategies.

Users may view, print, copy, and download text and data-mine the content in such documents, for the purposes of academic research, subject always to the full Conditions of use: http://www.nature.com/authors/editorial_policies/license.html\#terms

Corresponding Author: Marc Dall'Era, 4860 Y Street, Suite 3500, Sacramento, CA 95864, mdallera@ ucavis.edu, 916-734-2893.

Conflicts of Interest: Dr. Dall'Era has received compensation from Genomic Health for his roles as advisor and speaker. Dr. Klotz has no conflicts of interest to declare. 


\section{Introduction}

Active surveillance (AS) has gained widespread acceptance as the initial treatment choice for certain patients with prostate cancer. Up to $90 \%$ of contemporary low risk men are initially managed in this fashion.(1) Several factors have contributed to increased utilization of active surveillance over the last decade, allowing many men to avoid unnecessary interventions and treatment related side effects. Multiple ongoing series have collected prospective data on men managed expectantly, utilizing varied selection criteria and surveillance strategies. These series, some with long-term follow-up, have documented that an approach of initial surveillance with delayed treatment after risk re-stratification can be safe and effective. In addition, an emphasis on reducing over-treatment of prostate cancer has increased in response to the United States Preventative Service Task Force's recommendation against widespread PSA screening in 2009.(2)

When discussing AS, patients are often placed into risk categories based on known clinical features as defined by D'Amico criteria, the National Comprehensive Cancer Network (NCCN), or Cancer of the Prostate Risk Assessment (CAPRA) scoring algorithm.(2-4) Although most published data with AS has focused on men with very low and low risk prostate cancer, patients and practitioners continue to question selection criteria for this approach. Some have advocated expanding these to include men with intermediate risk features. The purpose of this article is to review the background and rational for AS, and summarize the experience with this approach for men with intermediate risk prostate cancer.

\section{Materials and Methods}

We queried Pubmed for all relevant original publications describing outcomes for men with prostate cancer managed with active surveillance. Referenced studies must have defined patient inclusion criteria and surveillance strategies to identify early signs of disease progression when treatment with curative intent may be offered. Outcomes for patients with intermediate risk features as defined by the primary investigators were studied when available and compared with similar risk men undergoing immediate treatment.

\section{Results}

\section{Long-term Outcomes of active surveillance for Predominantly Low Risk Prostate Cancer}

Data from two large prospectively studied AS cohorts have recently been published from distinct North American populations. Klotz et al have reported an update on their series from the University of Toronto after a median follow up of 6.4 years (0.2-19.8) with 206 out of 993 men followed for over 10 years.(5) This included a heterogeneous group of men, $21 \%$ of whom were intermediate risk at diagnosis. For surveillance, PSA was monitored every 3 months for 2 years, then every 6 months. Repeat biopsy was performed within the first year and then every 3-4 years. Two hundred and sixty seven (27\%) men ultimately received definitive treatment to the prostate. Overall survival at 10 and 15 years was $80 \%$ and $62 \%$, respectively with 15 deaths (1.5\%) attributed to prostate cancer and 13 men alive with documented metastatic disease. Although $44 \%$ of the men who eventually developed 
metastatic disease presented with Gleason $3+4$ disease, $26 \%$ presented with clinical features of very low risk prostate cancer.

An update from a separate yet similarly large series from Johns Hopkins University described results utilizing more stringent selection criteria and surveillance strategies. Inclusion criteria for this study included only men with very low risk disease defined as Gleason $3+3$, PSA $\leq 10$, clinical stage T1c, and $\_$positive biopsy cores. Disease monitoring included yearly prostate biopsy for tumor grade or volume increase. With a median follow up of 5 years, 15-year estimated cancer specific and metastases free survival are 99.9 and $99.4 \%$, respectively. These two studies utilizing different inclusion criteria and management strategies demonstrate somewhat varying, yet promising long-term results, even with expanded selection criteria for AS. They also suggest that baseline risk assessment is imperfect and that certain men outside of the confines of traditionally low risk disease may be safely managed with surveillance and delayed intervention as indicated.

\section{Rationale for Expanded Patient Selection Criteria for Active Surveillance: Outcomes from immediate treatment of intermediate risk disease}

Cancer specific survival for the men in the studies above closely mirror results published with immediate radical intervention. Patients and significant others need to understand that risks of cancer progression and mortality with treatment may be low, but are not zero. In a multi-center series of over 12,000 men, estimated prostate cancer mortality 15 years after radical prostatectomy (RP) ranged from 5-38\% for men with lowest and highest risk disease, respectively.(6) Men with Gleason score 7 disease experienced 10 and 15-year cancer specific mortality rates of $5 \%$ and $17 \%$, respectively after RP. Data from the National Prostate Cancer Register of Sweden on men diagnosed with localized, intermediate risk disease shows the cumulative prostate cancer specific mortality after 10 years was $3.4 \%$ after RP and 3.8\% after radiotherapy.(7) These rates, similar to the United States cohort at 10years, likely will increase with longer-term follow up. They are not significantly different than the outcome described by Klotz et al with AS.(5)

There is evidence that some prostate tumors with intermediate risk features may have indolent behaviors and be considered 'low-intermediate risk'. The highest rates of cancer related mortality after prostatectomy appear to be from Gleason grade 8-10 tumors and from those with seminal vesicle invasion. Therefore predicting these features based on pretreatment clinical features may be most critical when predicting long term outcome. (8) Predicting the presence of low risk tumors based on clinical features alone is not straightforward. Analyzing men with intermediate risk features who underwent immediate RP, Gandaglia et el identified potential predictors of organ confined, low grade cancer on final pathologic analysis.(9) Men were identified who had pre-operative clinical Gleason pattern 3+4 disease, and/or PSA 10-20 ng/ml, and/or clinical stage T2b-c. The primary outcomes were pathologically favorable disease at prostatectomy. Nineteen percent had diagnostic biopsy Gleason 6 tumors while 57\% had Gleason 3+4 and 23\% Gleason 4+3. Ten percent of the group had favorable pathologic features (organ confined, pathologic Gleason $3+3$ only) at the time of surgery. These men were more likely to be younger at diagnosis with lower PSA densities and biopsy Gleason score of 6 . Forty-one percent of the men with 
favorable RP pathology had biopsy Gleason 7 disease. Additionally, the men with favorable pathology had fewer numbers of positive cores at diagnosis and lower percentage of positive biopsy cores. These data suggest that men with some traditionally intermediate risk features including PSA $>10$ or Gleason $3+4$ tumors at diagnosis may actually harbor indolent tumors. Furthermore, these men may further be stratified by estimates of tumor volume on biopsy such as numbers (or percentage) of positive cores or biopsy core length involved with carcinoma. For AS, therefore, the concept of 'low-intermediate' risk may apply to men with small volume, organ confined Gleason $3+4$ cancers.

Does immediate treatment differentially impact survival for low and intermediate risk prostate cancer? Comparing treatment outcomes between men with low and intermediate risk cancer, Arvold et al showed that intermediate risk men treated with either RP or brachytherapy, had a low $(0.9 \%)$ cumulative PCSM after median follow-up of 4.8 years which was not significantly different from men treated with only low risk disease.(10) The authors noted a positive association with age and PCSM within the intermediate risk cohort, suggesting older men with intermediate risk disease experience higher death rates from prostate cancer than younger cohorts. In a larger group of men treated with initial brachytherapy, Raldow et al compared low risk men to men defined as having 'favorable intermediate risk' disease (Gleason pattern $3+4,<50 \%$ positive cores, and $<2$ intermediate risk factors, stage cT2b,c, PSA 10-20, Gleason score 7).(11) After median duration of 7.7 years there were no discernable differences in disease specific survival between the groups, suggesting that some men with low volume Gleason $3+4$ disease have similar outcomes to men with lower risk features after treatment. This was also observed in a surgical study comparing men having a single biopsy core of $3+4$ with men having only $3+3$ disease before RP which demonstrated no differences in likelihood of unfavorable pathology in screendetected men with prostate cancer.(12) Although disease specific outcomes for intermediate risk disease after treatment are very good, there is clear heterogeneity within this risk category suggesting that some of these men demonstrate risk profiles similar to men commonly offered AS today. This emphasizes the opportunity to expand AS criteria.

\section{Experiences with Active Surveillance in Patients with Expanded Selection Criteria}

The number of men with features of intermediate risk prostate cancer who are currently offered AS is unknown. Non-curative management is initially recorded for around $14 \%$ of men with intermediate risk disease features within the SEER and National Cancer Database, however these datasets are unable to distinguish AS with defined surveillance strategies from traditional watchful waiting or just deferred treatment to the prostate.(13) Within the Swedish Prostate Cancer Registry, however, active surveillance was recorded as initial treatment choice for $16 \%$ of intermediate risk men.(14) At this time, most intermediate risk men in the Western world are offered curative therapy at the time of diagnosis. Several investigators have published their experiences of surveillance with delayed intervention for men with intermediate risk disease. Different patient selection criteria and surveillance strategies are described. The 'intermediate risk' category is defined differently for each of the studies analyzed (Table 1). Another confounder is the modification of the Gleason scoring system over time. The International Society of Urologic Pathology (ISUP) update to prostate cancer grading in 2005 resulted in upgrading of many pattern 3s to pattern 4, or 6 to 
7.(15) Certain low-risk patients included in series prior to 2005 without contemporary pathologic review, therefore may actually be similar to contemporary men with Gleason $3+4$ tumors.

The outcomes from the 5 identified papers including men with intermediate risk disease are presented in Table 2. Within the Toronto series of 993 men with prostate cancer managed with AS, a group of men with intermediate risk features (defined as PSA 10-20 or Gleason score $3+4)$ were included.(5) Tumor histology primarily defined this risk category with $63 \%$ of men deemed intermediate risk (13\% of entire cohort) by harboring Gleason 7 disease. The remaining intermediate risk men had PSA levels $>10$ and $3 \%$ of the entire cohort had both Gleason 3+4 disease and PSA >10. With a median follow up of 6.4 years, $1.5 \%$ of the cohort died from prostate cancer and actuarial CSS at 15 years was $95 \%$. On multivariate analysis, both diagnostic Gleason score and PSA were associated with overall survival and $44 \%$ of the men who progressed to metastatic disease had Gleason 7 at baseline while the rest were Gleason 6 . In a subsequent publication specifically describing the men who eventually developed metastatic disease, the authors note that the men considered intermediate risk at baseline due to PSA over 10 in the setting of Gleason 6 histology were not associated with increased risk for metastatic disease compared with the entire cohort. (16) No patients with surgically confirmed Gleason 6 progressed to metastatic disease, and all but 2 patients were upgraded prior to the development of metastases. These 2 men were both radiated for rapid PSA rise. These data demonstrate that overall metastases free and cancer specific survival for the cohort are high. Importantly, however, the presence of pattern 4 conferred a higher likelihood of progression to metastatic disease, despite these patients being otherwise favorable. In this study, patients were defined as having disease progression and offered additional therapy for PSA doubling time of $\mathfrak{B}$ years, histologic upgrade on repeat prostate biopsy, or clinical evidence of progression. Treatment was also associated with baseline PSA levels and Gleason score at 1 year, suggesting that intermediate risk men are more likely to receive intervention over time.

Cooperberg et al published early outcomes for men with traditional intermediate risk features managed with AS at the University of California, San Francisco.(17) Intermediate risk in this study was primarily defined by having Cancer of the Prostate Risk Assessment (CAPRA) scores of 3-5. Thirty percent of men in this group had Gleason 3+4 disease while $2 \%$ were Gleason $4+3$ at diagnosis. These men with intermediate risk features tended to be older at diagnosis (median age 65) than men with low risk disease. The median PSA for the overall cohort was 10.3 (range 3.14-37.91), suggesting that PSA levels drove risk category in many men more than histology. Clinical progression for this study was defined as Gleason upgrade to any $\geq 4+3$, PSADT $\_$or 3 years, and receipt of treatment. At 4 years, $61 \%$ of intermediate risk men were free from clinical progression, which did not differ significantly from men presenting with only low risk features and managed with AS at this institution. Furthermore, of 16 men who presented with intermediate risk disease and eventually were treated with RP, 50\% showed evidence of pathologic T3 disease, however none had positive lymph node metastases. In the short term, AS for men with intermediate risk features provided similar outcomes compared with lower risk men with selective delayed intervention for men with evidence for disease reclassification. 
Within the ERSPC dataset, a cohort of men having intermediate risk disease features and managed with AS was identified with reported long-term outcomes.(18) In this series, low risk was defined by the entry criteria for the Prostate Cancer Research International: Active Surveillance (PRIAS) protocol: cT1c/T2, PSA $₫$ 0, Gleason score $₫$, PSA density $₫ \mathbf{0} .2$ and $\mathcal{s}$ positive cores. Intermediate risk, therefore, consisted of men with PSA 10-20, and/or Gleason 7, and/or $\geq 3$ positive cores. Of 128 men with intermediate risk features, 28 had Gleason score of 7, while 77\% percent of men were Gleason 6 disease and considered intermediate risk for $>2$ positive cores or higher PSA density. For the intermediate risk cohort, 10-year estimated disease specific survival was $96.1 \%$ with $53.9 \%$ of the men undergoing deferred therapy. There were 5 deaths due to prostate cancer during the study period only two of which occurred in men presenting in the intermediate risk category. Although the intermediate risk men experienced lower treatment free and overall survival, there were no differences in disease specific mortality between the groups. Although the absolute numbers are small (1 vs. 3), 10-year metastases free survival was worse in men initially with intermediate risk disease $(96.4 \%$ vs. 99.7 for low risk, $\mathrm{p}=0.03)$. A separate analysis from this cohort of intermediate risk men all defined by having Gleason 7 disease estimated no disease specific deaths at 6 years with $26 \%$ of men receiving further treatment. (19) Treatment free survival was substantially higher for men who otherwise would have met clinical criteria for AS under the PRIAS protocol except for Gleason score. This demonstrates how measures of disease risk may bear different weights in risk assessment. No men who started with Gleason $4+3$ disease remained untreated at 7 years.

Within the series from The Royal Marsden Hospital, 88 (18\%) men were included with intermediate rise disease, 33 of who had Gleason 3+4 cancer.(20) After a median follow up of 5.7 years, $31 \%$ had received prostate directed treatments. On multivariate analysis, diagnostic Gleason score of 7 was associated with time to adverse histology on repeat prostate biopsy, defined by primary Gleason score $\geq 4+3$ or $>50 \%$ total prostate cores with cancer on repeat biopsy. Over this time period there were only 2 deaths attributed to prostate cancer, one of which occurred in a patient presenting with intermediate risk disease.

The Prostate Cancer Active Surveillance Study (PASS) is a prospective multicenter active surveillance study sponsored by the Canary Foundation.(21) This multicenter, prospective active surveillance study includes 115 men with intermediate risk disease by NCCN criteria (13\% of cohort), 56 of which have Gleason 3+4 disease. After a median follow up period of 28 months, $24 \%$ of the entire cohort experienced disease reclassification defined by higher Gleason grade or tumor volume on repeat biopsy. No association was noted with baseline NCCN risk category and either disease reclassification or adverse pathology after RP for those men treated with surgery. More specifically, $40 \%$ of men with intermediate risk disease at diagnosis had adverse pathology (defined as primary Gleason 4 or 5, extraprostatic extension, seminal vesicle invasion, or lymph node metastases) at RP. This did not differ significantly from men with baseline very low (37\%) or low (32\%) risk disease.

These studies demonstrate that AS is feasible for select men with prostate cancer and some intermediate risk features, and caution must be exercised in men with Gleason pattern 4 disease. These findings are important when making treatment decisions and counseling me with localized prostate cancer on options for therapy including AS. 


\section{Overcoming Barriers to Active Surveillance for Intermediate Risk Disease}

Treatment decisions for clinically localized prostate cancer are predominately based on risk estimations of disease progression and cancer specific mortality over time. Physicians must also consider competing risks of mortality from patient co-morbidities when counseling them on treatment options for localized prostate cancer. Patients with limited life expectancies ( $<10$ years) stand to benefit the least from immediate prostate directed therapy, however this is often challenging to estimate. Tools exist to help predict the long-term probability of non-cancer mortality for shared decision-making.(22) A number of clinical tools including classification systems, nomograms and risk calculators are available to estimate disease outcomes by considering multiple clinical variables, with disease histology being a predominant predictor. Clinical risk assessment based on a single set of prostate biopsies, however, may be underestimated, with a $23-46 \%$ likelihood of missing higher grade or higher stage tumors after a single biopsy session.(23) These figures are based on multiple surgical series comparing pathologic findings after radical prostatectomy from men who would have been considered candidates for surveillance based on contemporary definitions of very low and low risk disease and may be more pronounced for men with intermediate risk features.(23-26) Pathologic inter-observer variability is also well documented and may contribute to this histology tumor grade discrepancy at diagnosis and after repeat prostate biopsy.(27) Most changes, however, are fairly minimal. The commonest upgrading is from Gleason $3+3$ to Gleason 3+4. Likely much of the short-term data on AS comparing low and intermediate risk men reflects this inherent clinical under-sampling with standard ultrasound guided prostate biopsy.

For intermediate risk men additional biopsy features may be useful to define a 'lowintermediate' risk category. A recent analysis utilizing data from the Surveillance, Epidemiology, and End Results program (SEER) showed that $27.6 \%$ of men with clinical intermediate risk prostate cancer might actually have pathologic high-risk disease (T3-4 or Gleason 8-10).(28) They were able to further sub stratify the intermediate risk category into favorable or unfavorable intermediate risk by percentage of biopsy cores involved with carcinoma. Intermediate risk men with $\mathbf{5 0 \%}$ cores positive had $18.2 \%$ occult high risk disease while men with $\geq 50 \%$ positive cores had $34.2 \%$ risk. When considering AS, men with intermediate risk features will likely need to be further stratified and reserved for those with lower intermediate risk features (a minority of positive biopsy cores, only Gleason $3+4)$. Several studies suggest that PSA levels $>10$ as the sole intermediate risk factor may not correlate with higher risk for adverse pathology or biochemical recurrence after RP in the face of low grade disease especially when PSA density is considered. $(29,30)$ The latest publication from ISUP recommends that pathologists record the percent of Gleason pattern 4 for patients with $3+4$ disease which may help risk stratify men with intermediate risk features for AS.(31)

A further consideration is the significance of very small proportion of Gleason 4 in men with Gleason 7 cancer. A study by Huang et al reported that in those patients with $<=5 \%$ Gleason 4 pattern on biopsy, the distribution of radical prostatectomy grade was identical to the patients with Gleason 3+3. Undoubtedly tangential cut of a Gleason 3 acinus, which misses the lumen, may give the appearance of Gleason 4 pattern, and is responsible for artifactual 
upgrading in many of these cases, particularly where tumor volume is small. Thus patients with $<=5 \%$ Gleason 4 should be considered excellent surveillance candidates.(32) Intermediate risk patients with low PSA density appear to behave like low risk patients. Advances in risk assessment utilizing molecular testing and innovative imaging to predict and identify cancers with higher grade or volume upfront will help limit risk and expand patient selection criteria for AS.

\section{Novel Biomarkers}

Novel biomarkers are being developed and evaluated to improve patient selection for active surveillance. Many of these can be incorporated with standard clinical variables. In the setting of clinical intermediate risk features, these are an attractive tool to disaggregate disease heterogeneity based on clinical features alone. A 31-gene expression assay (Prolaris ${ }^{\circledR}$ ), which generates a cell cycle progression (CCP) score has been validated to predict PCSM in a heterogeneous group of conservatively, treated men.(33) Per unit increase in CCP, the Hazard Ratio (HR) for prostate cancer mortality for men with Gleason 3+4 cancer was 1.77 (95\%CI 1.22-2.57) and for men with Gleason 4+3 was 2.16 (95\%CI 1.31-3.56). Clinical risk prediction in this cohort utilizing the CAPRA score alone yielded a 10 -year prostate cancer mortality estimate of $4 \%$ for men with low risk disease. Forty-four percent of men with intermediate risk (CAPRA score $=3$ ) disease, however, had a predicted 10 -year PCSM $<4 \%$ when the CCP score was added to the clinical prediction model.

A 17-gene expression panel (Oncotype $\mathrm{Dx}^{\circledR}$ ) was designed to predict adverse pathologic features at the time of cancer diagnosis for men with National Comprehensive Cancer Network (NCCN) very low, low and low-intermediate risk disease.(34) Genes within this panel were selected based on the ability to predict outcomes regardless of tumor sampling. After incorporation of the Genomic Prostate Score (GPS), approximately $8 \%$ of men with NCCN intermediate risk features had predicted adverse pathology similar to men with NCCN low risk disease. In a separate validation study, the GPS was associated with adverse pathologic features at prostatectomy with an odds ratio per 20 point GPS increase of 3.3 (95\% CI 2.14-4.97).(35) These biomarkers are two examples with several others under investigation to help select candidates for active surveillance and potentially follow them over time.(36)

\section{Germline genetic variants/Single Nucleotide Polymorphisms (SNPs)}

Patient germline and somatic molecular data also may facilitate the identification of the best candidates for this approach . Prostate cancer family history and 39 previously identified prostate cancer SNPs were studied for their association with adverse outcomes (defined as adverse histology on repeat biopsy or receipt of treatment) within 471 men enrolled in an AS study at the Royal Marsden Hospital in England.(37) There was no association between having a first or second-degree relative with prostate cancer and either time to adverse histology or subsequent treatment. The authors also noted no association between the studied SNPs and these outcomes. Kearns et al studied the association of 23 SNPs with Gleason upgrading over time in North American surgical and AS cohorts of men with verylow and low risk prostate cancer.(38) After adjusting for multiple comparisons, a single SNP on chromosome 11q22 was associated with Gleason upgrading at the time of RP was also 
associated with eventual Gleason upgrading for men on AS undergoing repeat prostate biopsies over time. A separate analysis of $>242,000$ SNPs in primarily low risk Korean men undergoing prostatectomy also identified 15 alleles associated with higher Gleason grade disease at the time of surgery, one of which on chromosome 3 remained significant after adjusting for multiple testing.(39) These three studies from disparate countries and populations demonstrate the complexity of utilizing germline genetic variants to predict higher-grade disease or disease progression over time for surveillance of prostate cancer. Ongoing association studies including men with intermediate risk features may provide additional tools for expanding patient selection and surveillance strategies.

\section{Novel Imaging}

Most active surveillance protocols include repeat prostate biopsy overtime to limit the risk of disease misclassification and to monitor for disease progression. Trans-rectal prostate ultrasonography alone, however, is poorly sensitive for detecting early changes in tumor volume or grade when intervention may be recommended.(40, 41) Multi-parametric magnetic resonance imaging (mp-MRI) has emerged as the modality of choice for prostate cancer imaging. Diffusion weighted imaging sequences are particularly important and the apparent diffusion coefficient has proved useful for detecting primarily higher-grade prostate tumors. In the pre-diagnostic setting, mp-MRI with a technique for image fusion targeted biopsy is shown to improve detection of primarily higher-grade (Gleason $\geq 4+3$ ) tumors and has been corroborated in multiple studies.(42) Taken together, these data suggest usefulness in men on AS in order to reduce undergrading at the time of diagnosis as well as to monitor disease progression with time.

In a study of serial mp-MRI for men on active surveillance, Diaz et al demonstrated a relatively high negative predictive value (80\%) for changes in Gleason grade.(43) Men in this study were all low risk at study entry, however and therefore not directly applicable to men with intermediate features. No published studies to date report on the usefulness of mpMRI for performing active surveillance in men with intermediate risk features.

\section{Conclusions}

In the absence of clear and optimal selection criteria, patient specific goals and priorities of therapy must be considered and individualized for making treatment decisions and discussing how best to offer and perform AS for an expanded population of men with clinically localized prostate cancer. The contemporary experiences of surveillance for men with intermediate risk features suggest that although these men are at higher risk for eventually having additional therapy, they are not significantly compromising chances for longer-term cure. Men with more than minimal Gleason pattern 4, however, must be carefully selected and surveyed for early signs of progression and may be at increased risk of metastases. Information from advanced imaging and biomarker technology will likely individualize future treatment decisions while improving overall surveillance strategies for men with intermediate risk prostate cancer and should be assessed within a formal clinical trial. 


\title{
Acknowledgments
}

\author{
none \\ Source of funding: none
}

\section{References}

1. Cooperberg MR, Carroll PR. Trends in Management for Patients With Localized Prostate Cancer, 1990-2013. Jama. 2015; 314(1):80-2. [PubMed: 26151271]

2. Moyer VA. Force USPST. Screening for prostate cancer: U.S. Preventive Services Task Force recommendation statement. Annals of internal medicine. 2012; 157(2):120-34. [PubMed: 22801674]

3. Hernandez DJ, Nielsen ME, Han M, Partin AW. Contemporary evaluation of the D'amico risk classification of prostate cancer. Urology. 2007; 70(5):931-5. [PubMed: 18068450]

4. Cooperberg MR, Freedland SJ, Pasta DJ, Elkin EP, Presti JC Jr, Amling CL, et al. Multiinstitutional validation of the UCSF cancer of the prostate risk assessment for prediction of recurrence after radical prostatectomy. Cancer. 2006; 107(10):2384-91. [PubMed: 17039503]

5. Klotz L, Vesprini D, Sethukavalan P, Jethava V, Zhang L, Jain S, et al. Long-term follow-up of a large active surveillance cohort of patients with prostate cancer. Journal of clinical oncology : official journal of the American Society of Clinical Oncology. 2015; 33(3):272-7. [PubMed: 25512465]

6. Stephenson AJ, Kattan MW, Eastham JA, Bianco FJ Jr, Yossepowitch O, Vickers AJ, et al. Prostate cancer-specific mortality after radical prostatectomy for patients treated in the prostate-specific antigen era. Journal of clinical oncology : official journal of the American Society of Clinical Oncology. 2009; 27(26):4300-5. [PubMed: 19636023]

7. Stattin P, Holmberg E, Johansson JE, Holmberg L, Adolfsson J, Hugosson J, et al. Outcomes in localized prostate cancer: National Prostate Cancer Register of Sweden follow-up study. Journal of the National Cancer Institute. 2010; 102(13):950-8. [PubMed: 20562373]

8. Eggener SE, Scardino PT, Walsh PC, Han M, Partin AW, Trock BJ, et al. Predicting 15-year prostate cancer specific mortality after radical prostatectomy. The Journal of urology. 2011; 185(3):869-75. [PubMed: 21239008]

9. Gandaglia G, Schiffmann J, Schlomm T, Fossati N, Moschini M, Suardi N, et al. Identification of pathologically favorable disease in intermediate-risk prostate cancer patients: Implications for active surveillance candidates selection. The Prostate. 2015; 75(13):1484-91. [PubMed: 26177942]

10. Arvold ND, Chen MH, Moul JW, Moran BJ, Dosoretz DE, Banez LL, et al. Risk of death from prostate cancer after radical prostatectomy or brachytherapy in men with low or intermediate risk disease. The Journal of urology. 2011; 186(1):91-6. [PubMed: 21571341]

11. Raldow AC, Zhang D, Chen MH, Braccioforte MH, Moran BJ, D'Amico AV. Risk Group and Death From Prostate Cancer: Implications for Active Surveillance in Men With Favorable Intermediate-Risk Prostate Cancer. JAMA oncology. 2015; 1(3):334-40. [PubMed: 26181182]

12. Schiavina R, Borghesi M, Brunocilla E, Romagnoli D, Diazzi D, Giunchi F, et al. The biopsy Gleason score $3+4$ in a single core does not necessarily reflect an unfavourable pathological disease after radical prostatectomy in comparison with biopsy Gleason score 3+3: looking for larger selection criteria for active surveillance candidates. Prostate cancer and prostatic diseases. 2015; 18(3):270-5. [PubMed: 26055663]

13. Weiner AB, Patel SG, Etzioni R, Eggener SE. National trends in the management of low and intermediate risk prostate cancer in the United States. The Journal of urology. 2015; 193(1):95102. [PubMed: 25106900]

14. Loeb S, Berglund A, Stattin P. Population based study of use and determinants of active surveillance and watchful waiting for low and intermediate risk prostate cancer. The Journal of urology. 2013; 190(5):1742-9. [PubMed: 23727309]

15. Epstein JI, Allsbrook WC Jr, Amin MB, Egevad LL, Committee IG. The 2005 International Society of Urological Pathology (ISUP) Consensus Conference on Gleason Grading of Prostatic 
Carcinoma. The American journal of surgical pathology. 2005; 29(9):1228-42. [PubMed: 16096414]

16. Yamamoto T, Musunuru B, Vesprini D, Zhang L, Ghanem G, Loblaw A, et al. Metastatic Prostate Cancer in Men Initially Treated with Active Surveillance. The Journal of urology. 2015

17. Cooperberg MR, Cowan JE, Hilton JF, Reese AC, Zaid HB, Porten SP, et al. Outcomes of active surveillance for men with intermediate-risk prostate cancer. Journal of clinical oncology : official journal of the American Society of Clinical Oncology. 2011; 29(2):228-34. [PubMed: 21115873]

18. Bul M, van den Bergh RC, Zhu X, Rannikko A, Vasarainen H, Bangma CH, et al. Outcomes of initially expectantly managed patients with low or intermediate risk screen-detected localized prostate cancer. BJU international. 2012; 110(11):1672-7. [PubMed: 22928973]

19. van den Bergh RC, Roemeling S, Roobol MJ, Aus G, Hugosson J, Rannikko AS, et al. Gleason score 7 screen-detected prostate cancers initially managed expectantly: outcomes in 50 men. BJU international. 2009; 103(11):1472-7. [PubMed: 19154509]

20. Selvadurai ED, Singhera M, Thomas K, Mohammed K, Woode-Amissah R, Horwich A, et al. Medium-term outcomes of active surveillance for localised prostate cancer. European urology. 2013; 64(6):981-7. [PubMed: 23473579]

21. Newcomb LF, Thompson IM Jr, Boyer HD, Brooks JD, Carroll PR, Cooperberg MR, et al. Outcomes of Active Surveillance for the Management of Clinically Localized Prostate Cancer in the Prospective, Multi-Institutional Canary PASS Cohort. The Journal of urology. 2015

22. Daskivich TJ, Kwan L, Dash A, Saigal C, Litwin MS. An Age Adjusted Comorbidity Index to Predict Long-Term, Other Cause Mortality in Men with Prostate Cancer. The Journal of urology. 2015; 194(1):73-8. [PubMed: 25623745]

23. Conti SL, Dall'era M, Fradet V, Cowan JE, Simko J, Carroll PR. Pathological outcomes of candidates for active surveillance of prostate cancer. The Journal of urology. 2009; 181(4):162833. discussion 33-4. [PubMed: 19233388]

24. Smaldone MC, Cowan JE, Carroll PR, Davies BJ. Eligibility for active surveillance and pathological outcomes for men undergoing radical prostatectomy in a large, community based cohort. The Journal of urology. 2010; 183(1):138-43. [PubMed: 19913808]

25. Tosoian JJ, JohnBull E, Trock BJ, Landis P, Epstein JI, Partin AW, et al. Pathological outcomes in men with low risk and very low risk prostate cancer: implications on the practice of active surveillance. The Journal of urology. 2013; 190(4):1218-22. [PubMed: 23643603]

26. El Hajj A, Ploussard G, de la Taille A, Allory Y, Vordos D, Hoznek A, et al. Analysis of outcomes after radical prostatectomy in patients eligible for active surveillance (PRIAS). BJU international. 2013; 111(1):53-9. [PubMed: 22726582]

27. Thomsen FB, Marcussen N, Berg KD, Christensen IJ, Vainer B, Iversen P, et al. Repeated biopsies in patients with prostate cancer on active surveillance: clinical implications of interobserver variation in histopathological assessment. BJU international. 2015; 115(4):599-605. [PubMed: 24903618]

28. Dinh KT, Muralidhar V, Mahal BA, Chen YW, Nezolosky MD, Beard CJ, et al. Occult High-Risk Disease in Clinically Low-Risk Prostate Cancer with >/=50\% Positive Biopsy Cores: Should National Guidelines Stop Calling Them Low-Risk? Urology. 2015

29. Yu J, Kwon YS, Kim S, Han CS, Farber N, Kim J, et al. Pathological Outcome Following Radical Prostatectomy in Men with PSA Greater than 10 and Histologically Favorable-Risk Prostate Cancer. The Journal of urology. 2015

30. Faisal FA, Sundi D, Pierorazio PM, Ball MW, Humphreys EB, Han M, et al. Outcomes of men with an elevated prostate-specific antigen (PSA) level as their sole preoperative intermediate- or high-risk feature. BJU international. 2014; 114(6b):E120-9. [PubMed: 24731026]

31. Epstein JI, Egevad L, Amin MB, Delahunt B, Srigley JR, Humphrey PA, et al. The 2014 International Society of Urological Pathology (ISUP) Consensus Conference on Gleason Grading of Prostatic Carcinoma: Definition of Grading Patterns and Proposal for a New Grading System. The American journal of surgical pathology. 2016; 40(2):244-52. [PubMed: 26492179]

32. Huang CC, Kong MX, Zhou M, Rosenkrantz AB, Taneja SS, Melamed J, et al. Gleason score 3 $+4=7$ prostate cancer with minimal quantity of gleason pattern 4 on needle biopsy is associated 
with low-risk tumor in radical prostatectomy specimen. The American journal of surgical pathology. 2014; 38(8):1096-101. [PubMed: 24832163]

33. Cuzick J, Stone S, Fisher G, Yang ZH, North BV, Berney DM, et al. Validation of an RNA cell cycle progression score for predicting death from prostate cancer in a conservatively managed needle biopsy cohort. British journal of cancer. 2015; 113(3):382-9. [PubMed: 26103570]

34. Klein EA, Cooperberg MR, Magi-Galluzzi C, Simko JP, Falzarano SM, Maddala T, et al. A 17gene assay to predict prostate cancer aggressiveness in the context of Gleason grade heterogeneity, tumor multifocality, and biopsy undersampling. European urology. 2014; 66(3):550-60. [PubMed: 24836057]

35. Cullen J, Rosner IL, Brand TC, Zhang N, Tsiatis AC, Moncur J, et al. A Biopsy-based 17-gene Genomic Prostate Score Predicts Recurrence After Radical Prostatectomy and Adverse Surgical Pathology in a Racially Diverse Population of Men with Clinically Low- and Intermediate-risk Prostate Cancer. European urology. 2015; 68(1):123-31. [PubMed: 25465337]

36. Leapman MS, Nguyen HG, Cooperberg MR. Clinical Utility of Biomarkers in Localized Prostate Cancer. Current oncology reports. 2016; 18(5):30. [PubMed: 27023445]

37. Goh CL, Saunders EJ, Leongamornlert DA, Tymrakiewicz M, Thomas K, Selvadurai ED, et al. Clinical implications of family history of prostate cancer and genetic risk single nucleotide polymorphism (SNP) profiles in an active surveillance cohort. BJU international. 2013; 112(5): 666-73. [PubMed: 23320731]

38. Kearns JT, Lapin B, Wang E, Roehl KA, Cooper P, Catalona WJ, et al. Associations Between iCOGS Single Nucleotide Polymorphisms and Upgrading in Both Surgical and Active Surveillance Cohorts of Men with Prostate Cancer. European urology. 2016; 69(2):223-8. [PubMed: 26411806]

39. Oh JJ, Park S, Lee SE, Hong SK, Lee S, Choe G, et al. The use of exome genotyping to predict pathological Gleason score upgrade after radical prostatectomy in low-risk prostate cancer patients. PloS one. 2014; 9(8):e104146. [PubMed: 25093842]

40. Dall'Era MA, Konety BR, Cowan JE, Shinohara K, Stauf F, Cooperberg MR, et al. Active surveillance for the management of prostate cancer in a contemporary cohort. Cancer. 2008; 112(12):2664-70. [PubMed: 18433013]

41. Washington SL, Bonham M, Whitson JM, Cowan JE, Carroll PR. Transrectal ultrasonographyguided biopsy does not reliably identify dominant cancer location in men with low-risk prostate cancer. BJU international. 2012; 110(1):50-5. [PubMed: 22077660]

42. Siddiqui MM, Rais-Bahrami S, Turkbey B, George AK, Rothwax J, Shakir N, et al. Comparison of $\mathrm{MR} / \mathrm{ultrasound}$ fusion-guided biopsy with ultrasound-guided biopsy for the diagnosis of prostate cancer. Jama. 2015; 313(4):390-7. [PubMed: 25626035]

43. Walton Diaz A, Shakir NA, George AK, Rais-Bahrami S, Turkbey B, Rothwax JT, et al. Use of serial multiparametric magnetic resonance imaging in the management of patients with prostate cancer on active surveillance. Urologic oncology. 2015; 33(5):202. e1-7. 

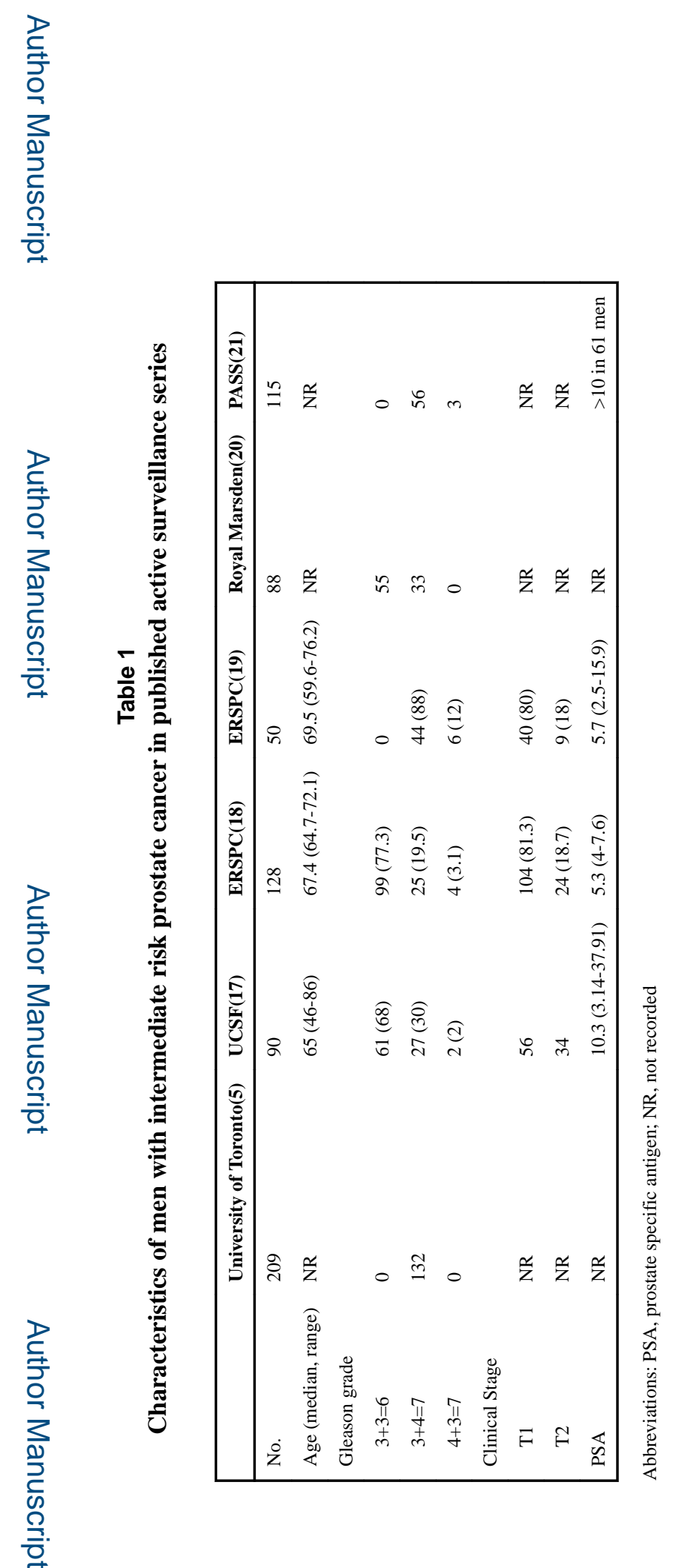

Prostate Cancer Prostatic Dis. Author manuscript; available in PMC 2017 May 01. 
Table 2

\section{Reported outcomes from active surveillance for intermediate risk men}

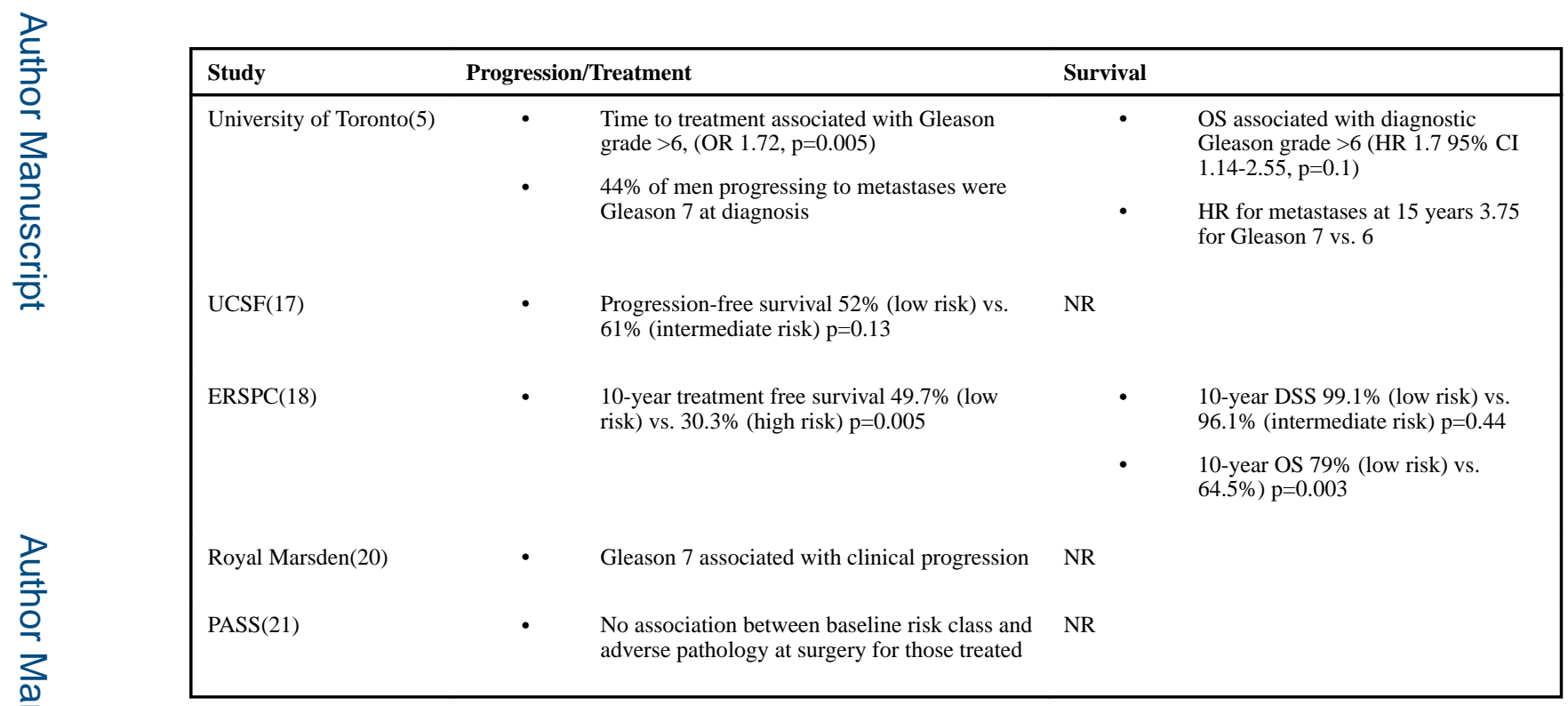

Abbreviations: OR, odds ratio; HR, hazard ratio; OS, overall survival; DSS, disease specific survival 